\title{
Comunicación
}

\section{PREVALENCIA DE Trypanosoma vivax EN BOVINOS DE SELVA ALTA EN LA PROVINCIA DE CHACHAPOYAS, AMAZONAS}

\author{
Manuel Tafur T. ${ }^{1}$, Amanda Chávez V., ${ }^{2}$, Eva Casas A. ${ }^{2}$ y Enrique Serrano M. ${ }^{2}$
}

\section{Abstract}

The aim of the present research was to determine the prevalence of Trypanosoma vivax in cattle of the province of Chachapoyas, located in the tropics of Perú. A total of 270 blood smears from 270 criollos, Holstein, Brown Swiss and Zebu crosses were evaluated. The prevalence of $T$. vivax was $10.0 \pm 3.4 \%$ (27/270) without differences between sexes $(P>0.05)$. None of the positive animals showed clinical signs related to trypanosomosis. The study reported for the first time the presence of $T$. vivax in the (Chachapoyas province. Further studies in this and other tropical regions using more sensitive techniques are recommended.

Key words: Trypanosoma, bovine, rain forest, Amazon, tropics

En base al informe de la Oficina de Información Agraria (1999) el Perú cuenta con una población de 4'903,363 cabezas de bovinos que constituyen una fuente importante de ingresos para el criador y la industria agropecuaria nacional. El $10.3 \%$ de la población bovina se encuentra en zona tropical, mayormente en la denominada selva alta, considerada como la mejor región agropecuaria del oriente peruano (Benavides, 1993; INEI, 2000).

La tripanosomosis es una enfermedad producida por diferentes especies del género Trypanosoma, el cual recibe diferentes denominaciones según sus características morfológicas, localización, nombre del descubridor, etc. Se encuentran parasitando la sangre y tejidos afectando a humanos y animales (Atías, 1994; Cordero del Campillo y Rojo, 1999). El T. congolense, T. vivax, T. evansi, T. brucei y T. theileri se encuentran ampliamente distribuidos en África afectando a bovinos y otros rumiantes. En América del Sur se han descrito el T. vivax, T. evansi y $T$. theileri en bovinos (Dávila y Aguilar, 2000; Muñoz y Chávez, 2001 y T. evansi y T. equiperdum en equinos (Dwinger y Hall, 2000).

En el Perú se ha diagnosticado la presencia de los hemoparásitos Babesia bovis, B. bigemina, T. vivax, y T. evansi (Rojas, 1990; Muñoz y Chávez, 2001). Sin embargo, no se cuentan con estudios concretos de prevalencia de estos hemoparásitos en las zonas tropicales y subtropicales del país (Alva, 1998). No obstante, se reconoce que el potencial agropecuario se encuentra limitado por la presencia de hemoprotozoarios en el ganado que pueden causar una muerte prematura o debilidad crónica (FAO, 1997). La

\footnotetext{
${ }^{1}$ Práctica privada

${ }^{2}$ Laboratorio de Microbiología y Parasitología, FMV-UNMSM

${ }^{3}$ E-mail: $a \_c h a v e z \_g @ h o t m a i l . c o m$
} 
tripanosomosis, al igual que sus vectores (tábanos y stomoxys), se encuentra ampliamente distribuida en toda América afectando la producción y rentabilidad de los sistemas de producción animal (Wells, 1984).

El diagnóstico del Trypanosoma vivax en bovinos al pastoreo no es simple cuando se encuentra en forma subclínica, y por ello es importante el monitoreo parasitológico para estimar su frecuencia de infección; de allí que se haya realizado el presente estudio a fin de determinar la prevalencia de Trypanosoma vivax en bovinos de la selva alta del Perú.

El trabajo fue realizado durante los meses de abril a junio del 2000 , en la provincia de Chachapoyas, ubicada al sur del departamento de Amazonas. La zona se encuentra a una altitud de $2,300 \mathrm{msnm}$, y presenta una topografía accidentada, con zonas de cordillera y valles interandinos, clima templado, moderadamente lluvioso y temperaturas que varían de $10^{\circ} \mathrm{Ca} 28^{\circ} \mathrm{C}$. La precipitación pluvial anual varía de 550 a $1,066 \mathrm{~mm}$, y presenta estaciones climáticas de seca entre mayo-agosto y lluviosa de setiembre a abril (INEI, 1985).

El tamaño de la muestra se calculó con la fórmula de proporciones en poblaciones finitas (Daniel, 1996) de acuerdo al 14\% de prevalencia encontrada en bovinos de la zona de Pucallpa (Calderón y Bazalar, 1978). El tamaño mínimo de la muestra fue de 186 , pero se trabajó con 270 bovinos para una mejor estimación.

Los animales muestreados tenían edades comprendidas entre 1 y 10 años, siendo la mayoría de ellos Bos taurus criollos cruzados con ganado Holstein, Brown Swiss y cebú en diferentes grados. Las muestras de sangre se colectaron en tubos vacutainer estériles y con EDTA. Se identificaron por número, sexo y procedencia. Se hicieron frotices en forma inmediata, y se fijaron con metanol durante dos minutos. La coloración con Giensa y la lectura de las láminas se hizo en el Laboratorio de Parasitología de la Facultad de Medicina Veterinaria de la Universidad Nacional Mayor de San Marcos.

La identificación del parásito se hizo a través de las características morfológicas y biométricas reportadas en la literatura, tales como el extremo posterior redondeado, flagelo libre, kinetoplasto grande y terminal, una membrana ondulante y una longitud corporal de 15 a 30 um (Soulsby, 1987; Dwinger y Hall, 2000).

Los resultados obtenidos en las pruebas fueron expresados en porcentajes de positividad teniendo en consideración la presencia del parásito en las láminas coloreadas con Giemsa. Se estableció el porcentaje de prevalencia con su respectivo intervalo de confianza al $95 \%$. La asociación entre sexo y presencia del parásito se evalúo a través de la prueba de Chi cuadrado (Daniel, 1996).

Estudios anteriores habían demostrado la presencia de T. vivax en selva baja (Calderón y Bazalar, 1978); sin embargo, este estudio demuestra por primera vez la presencia de T. vivax en la selva alta del Perú.

La prevalencia de T. vivax en 270 frotices sanguíneos de bovinos criollos de la provincia de Chachapoyas fue de $10.0 \pm$ $3.6 \%$. El sexo del animal no afectó la presentación de T. vivax (Cuadro 1).

Existen numerosos reportes sobre la presencia de $T$. vivax en diferentes áreas de Sudamérica. Así, en el Estado de Guarico, Venezuela, se encontró una prevalencia del 79.8\% en vacunos de doble propósito en tanto que en ganado altamente acebuado fue de 76.4\% (Tamasaukas y Roa, 1993). En 1995 se reportaron brotes de $T$. vivax en ganado seleccionado de Brasil, donde se encontró una prevalencia del $34 \%$; mientras que en Laguna Concepción, Bolivia, se halló el 86\% (Silva et al., 1996). Cabe destacar que el $T$. vivax es considerado actualmente endémico en las regiones tropicales vecinas como Co- 
Cuadro 1. Frecuencia de bovinos positivos a Trypanosoma vivax según sexo del animal, por el método parasicológico de frotís sanguíneo coloreado con Giemsa. Chachapoyas. Abril-junio 2000

\begin{tabular}{|c|c|c|c|}
\hline \multirow{2}{*}{ Sexo } & \multirow{2}{*}{$\begin{array}{c}\text { Muestras } \\
\text { (n) }\end{array}$} & \multicolumn{2}{|c|}{ Animales positivos } \\
\hline & & $\mathrm{n}$ & $\% \pm \mathrm{IC}^{1}$ \\
\hline Machos & 129 & 12 & $9.3 \pm 4.5$ \\
\hline Hembras & 141 & 15 & $10.6 \pm 5.2$ \\
\hline & 270 & 27 & $10.0 \pm 3.6$ \\
\hline
\end{tabular}

${ }^{1}$ Intervalo de confianza

lombia (Agudelo et al., 1984), en la región del Pantanal en Brasil y las tierras bajas en Santa Cruz, Beni, en Bolivia (Silva et al., 1996).

Las diferencias en el porcentaje de prevalencia del presente estudio con los resultados obtenidos en los países vecinos podrían deberse a las diferentes técnicas utilizadas. En esos trabajos se usaron las técnicas de IFI o ELISA, que son de mayor sensibilidad y especificidad que la realizada en nuestro estudio, la cual presenta una sensibilidad del 10\% (Botero, 1998).

Los animales positivos en este estudio no evidenciaron signos clínicos o síntomas de la enfermedad, encontrándose el hematocrito dentro de los rangos normales; de allí que se podría considerar que el $T$. vivax se encuentra en forma subclínica en la región de Chachapoyas.

En el presente estudio se puede concluir que la prevalencia de Trypanosoma vivax en bovinos de la provincia de Chachapoyas, Amazonas, mediante el examen de frotis sanguíneo coloreado con Giemsa fue $10.0 \pm 3.6 \%$. Se recomienda realizar estudios adicionales para determinar la prevalencia de $T$. vivax, en éstas y otras áreas ganaderas de clima tropical y subtropical del país, utilizando preferentemente técnicas de mayor sensibilidad y especificidad.

\section{Limeratura Ctrada}

1. Agudelo, M.T.; J.D. Mogollón; L.E. Torre; N.E. Peña; J. Barrera. 1984. Prevalencia de Trypanosoma vivax en bovinos de Villavicencio por pruebas parasitológicas directas y por inmunofluorescencia indirecta. Rev. Inst. Col. Agropec. 19: 33-37.

2. Alva, P.B. 1998. Seroprevalencia de Babesia bovis en bovinos del distrito de Oxapampa. Tesis Bach. Fac. Med. Vet., Univ. Nac. Mayor de San Marcos, Lima. $37 \mathrm{p}$.

3. Atías, A. 1994. Enfermedad de Chagas. $3^{a}$ ed. p 255-267. Ed. Mediterráneo. Santiago de Chile.

4. Benavides, A. 1993. Geografía del Perú. $2^{a}$ ed. p 71-73. Ed. Escuela Nueva. Lima.

5. Botero, D. 1998. Parasitosis humana. $3^{\text {a }}$ ed. p 203-432. Ed. Rojo. Medellín, Colombia.

6. Calderón, G.; H. Bazalar. 1978. Presencia de Trypanosoma vivax en ganado vacuno de Pucallpa. Boletín Soc. Per. Parasitología 1: 5.

7. Cordero de Campillo, M.; F.A. Rojo. 1999. Parasitología veterinaria. p 302309. Ed. McGraw-Hill Interamericana. España.

8. Daniel, W.W. 1996. Bioestadística. Base para el análisis de las Ciencias de la Salud. $5^{\mathrm{a}}$ ed. p 206. Noriega Editores. México. 
9. Dávila, M.R.; M.S. Aguilar. 2000. Animal trypanosomiasis in South America. Anny Acad. Sci. 216: 199-207.

10.Dwinger, R.H.; M.J.R. Hall. 2000. Trypanosomosis due to Trypanosoma vivax in ruminants in Latin America: a review. Animal Trypanosomosis: Diagnosis and Epidemiology. p 51-54. IAEA, Viena-Austria.

11. FAO. 1997. El costo de la tripanosomiasis. Rev. Española de Quimioterapia 1(1). Disponible: http//wwwmeds.../ tripanosomiosishtmyacom.

12. Instituto Nacional de Estadística (INEI). 1985. Resumen Estadístico Anual Departamental de la Dirección Regional Amazonas. Lima. Perú: $8 \mathrm{p}$.

13. Instituto Nacional de Estadística (INEI). 2000. Compendio Estadístico Económico Financiero. p 100-200. Ed. Monterrico. Lima.

14. Muñoz, K.; A. Chávez 2001. Trypanosoma evansi isopated from capybara (Hidrochaeris hidrochaeris). Hem. Inst. Oswaldo Cruz, Rio de Janeiro Brasil 96: 945-946.

15. Oficina de Información Agraria (OIA). 1999. Producción Pecuaria e Industria
Avícola del Perú. Resultados definitivos. p 144-148. Ministerio de Agricultura. Lima. Perú.

16. Rojas, C.M. 1990. Parasitismo de los rumiantes domésticos. p 302-306. Ed. MAIJOSA. Lima, Perú.

17. Silva, R.A.M.S.; J.A. Da Silva; R.C. Schneider; J. De Freitas; D. Mesquita; T. Mesquita; L. Ramírez; T.A.M.R. Dávila; M.E.B. Pereira. 1996. Outbreak of trypanosomiasis due to Trypanosoma vivax (Ziemann, 1905) in bovines of the Pantanal, Brazil. Mem. Inst. Oswaldo Cruz. 91: 561-562.

18. Soulsby E. 1987. Parasitología y enfermedades parasitarias en los animales domésticos. $7^{\mathrm{a}}$ ed. p 521. Ed. Interamericana. México.

19. Tamasaukas, R.; N. Roa. 1993. Prevalencia de la tripanosomiasis bovina ( $T$. vivax) en el Estado Guarico, Venezuela. XI Congreso Latinoamericano de Parasitología y I Congreso Peruano de Parasitología. Sociedad Peruana de Parasitología. p 133.

20. Wells, E.A. 1984. Animal tripanosomiasis in South América. Prev. Vet. Med. 2: 3141. 